\title{
Separation of variables in Hamilton-Jacobi and Klein-Gordon-Fock equations for a charged test particle in the Stackel spaces of type (1.1)
}

\author{
V. V. Obukhov
}

\begin{abstract}
All equivalence classes for electromagnetic potentials and space-time metrics of Stackel spaces, provided that Hamilton-Jacobi equation and Klein-Gordon-Fock equation for a charged test particle can be integrated by the method of complete separation of variables are found. The separation is carried out using the complete sets of mutually-commuting integrals of motion of type (1.1). Whereby in a privileged coordinate system the given equations turn into parabolic type equations. Hence, these metrics can be used as models for describing plane gravitational waves.
\end{abstract}

Keyword: Hamilton-Jakobi equation, separation of variables, Killings vectors and tensors, integrals of motion.

Tomsk State Pedagogical University, 60 Kievskaya St., Tomsk, 634041, Russia. Tomsk State University of Control Systems and Radioelectronics 40 Lenin Ave.,

Tomsk, 634050, Russia

\section{Introduction}

The main goal of this work is to complete the classification of Stackel spaces, started in [1] - 3. In the spaces the Hamilton-Jacobi equation for a charged test particle moving in an external electromagnetic field admits complete separation of variables. The theory of Stackel spaces is presented in [4 - 9]. A detailed bibliography can be found in [10] - [11].

According to the main theorem proved in [7] - 8], the Stackel space is characterized by a complete set of geometric objects. The set consists of mutually commuting Killing vector and tensor fields that satisfy some additional conditions, that allow carrying out complete separation of variables in classical and quantum equations of motion of a test particle in the presence of physical fields of different nature. A large 
number of works have been devoted to the corresponding classification problems. (see, for example, an overview in [2]). This article lists all non-equivalent cases of complete separation of variables of type (1.1).

The classification is carried out for the case when the complete set consists of two Killing tensor fields and one null Killing vector field without any additional restrictions on the electromagnetic field.

\section{Hamilton-Jakobi equation}

Let us present information from the theory of Stackel spaces which will be required further. Consider a $n$-dimensional Riemannian manifold $V_{n}$, with a coordinate system $\left\{x^{i}\right\} \quad(i, j, k, l=0, \ldots, n-1)$ and a metric tensor, with components $g_{i j}$ and $g^{i j}$.

Hereinafter $\delta^{i j}, \delta_{i j}, \delta_{j}^{i} \quad$ are Kronecker symbols, $\quad \lambda, \lambda_{i}=$ const (separation constants), $\quad \varepsilon, \varepsilon_{i} \quad$ can have values $0,+1,-1$.

Functions that depend only on the variable $x^{i}$ will be denoted by the lowercase letters with the obligatory lower index $i$, constants are marked with lowercase letters and a tilda sign. Everywhere in the text, there is a summation within the specified limits of index change on repeated upper and lower indexes.

Natural Hamilton-Jakobi equation (the term was suggested by Benenti in [12]) has the form:

$$
H=g^{i j} p_{i} p_{j}+2 A^{i} p_{i}+B=\lambda,
$$

where $p_{i}=\partial S / \partial x^{i}=S_{, i}$. Particular case of equation (11) is the regular HamiltonJakobi equation:

$$
H=g^{i j} p_{i} p_{j}+2 A^{i} p_{i}+A_{i} A^{i}=\lambda,
$$

Definition 1 The equation (11) allows a complete separation of variables if the space $V_{n}$ contains a privileged coordinate system $\left\{u^{i}\right\}$ in which the complete integral can be represented as:

$$
S=\sum_{i=0}^{n-1} s_{i}\left(u^{i}\right) .
$$

In this case, $\quad V_{n}$ is called Stackel space.

Definition 2 Let $V_{n} \quad$ has a set of geometric objects, consisting of mutuallycommuting $N<n$ Killing vector fields $-Y_{p}^{i}$ and $n-N \quad$ Killing tensor fields - $\quad X_{\nu}^{i j} \quad$ (including metric tensor: $\quad g^{i j}=X_{n-1}^{i j}$ ), satisfying the conditions: 
1) From $B^{\nu} X_{\nu}^{i j}+B^{p q} Y_{p}^{i} Y_{q}^{j}=0 \quad$ it follows $\quad B^{\nu}=B^{p q}=0$.

2) There are functions $B_{\mu \tau}^{\nu}, B_{\mu \nu}^{p q}, \quad B_{\mu q}^{p}, \quad$ such that

$$
\begin{gathered}
X_{\mu}^{i l} g_{l k} X_{\nu}^{k j}=B_{\mu \nu}^{\tau} X_{\tau}^{i j}+B_{\mu \nu}^{p q} Y_{p}^{i} Y_{q}^{j}, \\
X_{\mu}^{i l} g_{l k} Y_{p}^{k}=B_{\mu p}^{q} Y_{q}^{i} .
\end{gathered}
$$

3) There are functions, $B_{\nu}$ such that

$$
\operatorname{det} B_{\nu} B_{\mu \tau}^{\nu}>0 \text {. }
$$

In this case the set is called full. Hereinafter $\quad p, q=0, \ldots, N-1, \quad \nu, \mu, \tau=$ $N, \ldots, n-1$.

Let us denote

$$
N_{0}=N-\operatorname{rank}\left\|Y_{p}^{i} g_{i j} Y_{q}^{j}\right\| .
$$

If $N_{0} \neq 0$, the equation (1) is a parabolic one and among the non-ignored variables $u^{\nu}$ it can be distinguished as the variables $u^{\nu_{0}}, \quad\left(\nu_{0}, \mu_{0}=N, \ldots, N+\right.$ $N_{0}$ ) such that $g^{\nu_{0} \mu_{0}}=0$. It can be shown that if the space has the Lorentzian signature, the number $\quad N_{0} \quad$ can take one of the two values: 0 or 1 .

Theorem 1 The existence of a complete set is a necessary and sufficient condition for the complete separation of variables in the equation (11).

Definition 3 The space $V_{n}$, which allows a complete set is called Stackel space of $\left(n, N, N_{0}\right)$ type (or simply - of type $\left(N, N_{0}\right)$ for the case of space-time signature).

Theorem 2 If the equation (10) allows a complete separation of variables, the complete set of the motion integrals can be represented as

$$
\hat{X}_{q}=\delta_{q}^{i} p_{i}, \quad \hat{X}_{\mu}=\left(\hat{\phi}^{-1}\right)_{\mu}^{\nu}\left(\varepsilon_{\nu} p_{\nu}^{2}+2 h_{\nu}^{\nu q} p_{\nu} p_{q}+2 h_{\nu}^{q} p_{q}+2 h_{\nu}^{\nu} p_{\nu}+h_{\nu}\right) .
$$

where $Y_{p}^{i}=\delta_{p}^{i}$ are Killing vectors. Killing tensors can be represented in the form:

$$
X_{\mu}^{i j}=\left(\hat{\phi}^{-1}\right)_{\mu}^{\nu}\left(\delta_{\nu}^{i} \delta_{\nu}^{j} \varepsilon_{\nu}+\delta_{p}^{i} \delta_{q}^{j} h_{\nu}^{p q}+2 \delta_{p}^{i} \delta_{\nu}^{j} h_{\nu}^{p \nu}\right),
$$

where $\left(\hat{\phi}^{-1}\right)_{\mu}^{\nu}$ are elements of the matrix which is inverse of the Stackel matrix $\left\|\phi_{\nu}^{\mu}\right\|$. For the case of the regular equation (2) the following conditions are satisfied:

$$
A^{i}=\left(\hat{\phi}^{-1}\right)_{n-1}^{\nu} h_{\nu}^{i}, \quad A_{i} A^{i}=\left(\hat{\phi}^{-1}\right)_{n-1}^{\nu} h_{\nu},
$$


As it was noted above, in the space $V_{4}$ with the Lorentzian signature $N_{0}=0.1$. Therefore, there are seven disjoint types of Stackel space-times with the given signature. Respectively: four non-null types: $(0.0),(1.0),(2.0),(3.0)$; and three null types: (3.1), (2.1), (1.1). For the type (0.0) the electromagnetic potential $A_{i}=0$. In the privileged coordinate systems of space-times of type $\left(3 . N_{0}\right)$ there is only one non ignored variable. All components of the metric tensors and electromagnetic potentials depend on this variable and Hamilton-Jacobi equation (both and Klein-Gordon-Fock equation) admits complete separation of variables.

The classification of the space-time metrics of type (1.0) was carried out in [1, the classification of the space-time metrics of type (2.0) - in [2] and the classification of space-time metrics of type (2.1) - in [3]. Thus, it is only left to consider the spacetime metrics of type (1.1).

The metrics of the space-times of type (1.1) have been widely used by researchers in the study of various problems. For example, all privileged coordinate systems and all electromagnetic fields in which the Hamilton-Jacobi both and Klein-Gordon-Fock equations in flat space-time admit complete separation of variables of type (1.1) were found in the papers [13] - [15]. In the papers [16] - [17] the same problem was solved for vacuum both and electrovacuum Einstein equations, as well as in the Vaidya problem: (see [18]-20]). Separate classification problems were considered for the Einstein equations with dusty matter [21]-[22].

There is only one Killing vector field in the space-time of type (1.1): $\quad Y^{i}=\delta_{0}^{i}$. Thus $u^{0}$ is the ignored variable. As

$$
Y^{i} Y_{i}=0 \rightarrow g_{00}=0 \rightarrow g^{11}=0,
$$

equation (11) is parabolic. When considering an electromagnetic field, the presence of a zero non-ignored variable in a privileged coordinate system may mean that this field describes a plane electromagnetic wave. By analogy, some space-time metrics of the type (1.1) can be considered as a model of a plane gravitational wave. Let us mark the non-ignored variables with indexes $\alpha, \beta=1, \ldots, 3 ; \quad \nu, \mu=2,3$. Whereby $u^{1}$ is a null variable.

\subsection{Solution of the functional equation (6)}

Now the metric tensor and Killing tensor can be written in the privileged coordinate system as:

$$
\begin{array}{r}
X_{1}^{i j}=g^{i j}=\frac{1}{\Delta}\left(\left(\tau_{3}-\tau_{2}\right) h_{1}^{i j}+\left(\tau_{1}-\tau_{3}\right) h_{2}^{i j}+\left(\tau_{2}-\tau_{1}\right) h_{3}^{i j}\right), \\
X_{2}^{i j}=\frac{1}{\Delta}\left(\left(w_{2}-w_{3}\right) h_{1}^{i j}+\left(w_{3}-w_{1}\right) h_{2}^{i j}+\left(w_{1}-w_{2}\right) h_{3}^{i j}\right), \\
X_{3}^{i j}=\frac{1}{\Delta}\left(\left(w_{3} \tau_{2}-w_{2} \tau_{3}\right) h_{1}^{i j}+\left(\tau_{3} w_{1}-\tau_{1} w_{3}\right) h_{2}^{i j}+\left(\tau_{1} w_{2}-\tau_{2} w_{1}\right) h_{3}^{i j}\right),
\end{array}
$$


where

$$
\begin{gathered}
h_{\alpha}^{i j}=\left(\delta_{0}^{i} \delta_{1}^{j}+\delta_{0}^{j} \delta_{1}^{i}\right) \delta_{\alpha}^{1}+\delta_{\nu}^{i} \delta_{\nu}^{j} \delta_{\alpha}^{\nu} \varepsilon_{\nu}+\delta_{0}^{i} \delta_{0}^{j} b_{\alpha} . \\
\Delta=\left(\tau_{3}-\tau_{2}\right) w_{1}+\left(\tau_{1}-\tau_{3}\right) w_{2}+\left(\tau_{2}-\tau_{1}\right) w_{3}, \\
\left(\tau_{3}-\tau_{2}\right)=V^{1}, \quad\left(\tau_{1}-\tau_{3}\right)=V^{2}, \quad\left(\tau_{2}-\tau_{1}\right)=V^{3} .
\end{gathered}
$$

Hence, the equation (2) admits a complete separation of variables if the equation (66), the components of the metric tensor and electromagnetic potential have the form:

$$
\begin{gathered}
A^{\alpha} A_{\alpha}=\frac{\left(2 e_{1} a_{\alpha}-e_{1}^{2} b_{\alpha}\right) V^{\alpha}}{\Delta}=\frac{h_{\alpha} V^{\alpha}}{\Delta} . \\
\left(g^{i j}\right)=\left(\begin{array}{cccc}
\frac{b_{\alpha} V^{\alpha}}{\Delta} & \frac{V^{1}}{\Delta} & 0 & 0 \\
\frac{V^{1}}{\Delta} & 0 & 0 & 0 \\
0 & 0 & \frac{\varepsilon^{2} V^{2}}{\Delta} & 0 \\
0 & 0 & 0 & \frac{\varepsilon^{3} V^{3}}{\Delta}
\end{array}\right), \quad\left(g_{i j}\right)=\left(\begin{array}{cccc}
0 & \frac{\Delta}{V^{1}} & 0 & 0 \\
\frac{\Delta}{V^{1}} & -\frac{\Delta b_{\alpha} V^{\alpha}}{V^{2}} & 0 & 0 \\
0 & 0 & \frac{\varepsilon^{2} \Delta}{V^{2}} & 0 \\
0 & 0 & 0 & \frac{\varepsilon^{3} \Delta}{V^{3}}
\end{array}\right), \\
A^{i}=\frac{\delta^{i 0}\left(a_{\alpha} V^{\alpha}\right)+\delta^{i 1} e_{1} V^{1}}{\Delta}, \quad A_{i}=\delta_{i 0} e_{1}+\delta_{i 1} \frac{\left(a_{\alpha}-e_{1} b_{\alpha}\right) V^{\alpha}}{V^{1}}
\end{gathered}
$$

The functional equation (8) is reduced to a system of algebraic equations. In order to find all the metric tensors and electromagnetic potentials that allow to realize a complete separation of variables of type (1.1) in equation (2) it is necessary to solve this system. Note that using the acceptable transformations of coordinates and gradient transformations of the potential of the form

$$
u^{0} \rightarrow u^{0}+\int f_{1} d u^{1}, \quad A_{1} \rightarrow A_{1}+z_{1},
$$

one can turn the functions $\quad a_{1}$ and $b_{1}$ into zero. Then the equation (10) will be represent in the form:

$$
\left(2 e_{1} a_{2}-e_{1}^{2} b_{2}-h_{2}-h_{1}\right)\left(\tau_{3}-\tau_{1}\right)=\left(2 e_{1} a_{3}-e_{1}^{2} b_{3}-h_{3}-h_{1}\right)\left(\tau_{2}-\tau_{1}\right) .
$$

It is equivalent to the system of function equations:

$$
2 e_{1} a_{\nu}-e_{1}^{2} b_{\nu}-h_{\nu}-h_{1}=\theta_{1}\left(\tau_{\nu}-\tau_{1}\right) .
$$

The system has two solutions.

1) $\quad \dot{e}_{1} \neq 0 \rightarrow a_{\nu}=\tilde{a} t_{\nu}, \quad b_{\nu}=\tilde{b} t_{\nu}, \quad$ which is equivalent to: $\quad a_{\nu}=b_{\nu}=0$, where $\quad g^{00}=A^{0}=A_{1}=0$.

2) $e_{1}=\tilde{e}$, which is equivalent to: $e_{1}=0$. For both cases we have:

$$
A_{i} A^{i}=0 \quad \rightarrow h_{\alpha}=0
$$




\subsection{Results}

These solutions can be united. For this purpose let us introduce the symbols $\xi_{0}, \xi_{1}$ satisfying the following conditions:

$$
\xi_{0} \xi_{1}=0, \quad \xi_{0}+\xi_{1}=1 .
$$

Then the metric tensor and the electromagnetic potential will take the form:

$$
\begin{gathered}
\left(g^{i j}\right)=\left(\begin{array}{cccc}
\xi_{1} \frac{b_{\nu} V^{\nu}}{\Delta} & \frac{V^{1}}{\Delta} & 0 & 0 \\
\frac{V^{1}}{\Delta} & 0 & 0 & 0 \\
0 & 0 & \frac{\varepsilon^{2} V^{2}}{\Delta} & 0 \\
0 & 0 & 0 & \frac{\varepsilon^{3} V^{3}}{\Delta}
\end{array}\right), \quad\left(g_{i j}\right)=\left(\begin{array}{cccc}
0 & \frac{\Delta}{V^{1}} & 0 & 0 \\
\frac{\Delta}{V^{1}} & -\xi_{1} \frac{\Delta b_{\nu} V^{\nu}}{V^{12}} & 0 & 0 \\
0 & 0 & \frac{\varepsilon^{2} \Delta}{V^{2}} & 0 \\
0 & 0 & 0 & \frac{\varepsilon^{3} \Delta}{V^{3}}
\end{array}\right), \\
A^{i}=\frac{\delta^{i 0} \xi_{1}\left(a_{\nu} V^{\nu}\right)+\delta^{i 1} \xi_{0} e_{1} V^{1}}{\Delta}, \quad A_{i}=\delta_{i 0} \xi_{0} e_{1}+\frac{\delta_{i 1} \xi_{1}\left(a_{\nu} V^{\nu}\right)}{V^{1}}
\end{gathered}
$$

Functions $s_{\nu}$ from the equation (3) have the form:

$$
\begin{gathered}
s_{1}=\int \frac{\lambda_{1}+\lambda_{2} \tau_{1}+\lambda_{3} w_{1}}{2\left(\lambda_{0}+\xi_{0} e_{1}\right)} d u^{2}, \\
s_{\nu}=\varepsilon \int \sqrt{\varepsilon^{\nu}\left[\left(\lambda_{1}+\lambda_{2} \tau_{\nu}+\lambda_{3} w_{\nu}\right)-\xi_{1}\left(b_{\nu} \lambda_{0}^{2}+2 a_{\nu} \lambda_{0}\right)\right]} d u^{3}
\end{gathered}
$$

\section{Separation of variables in linear differential equations of the second order}

Let us consider the linear differential equation

$$
\hat{H} \Phi=\left(g^{i j} \hat{\partial}_{i} \hat{\partial}_{j}+W^{i} \hat{\partial}_{i}+W\right) \Phi=\lambda \Phi,
$$

where $\Phi, \quad W, \quad W^{i}$ are complex functions. The theory of variables separation in equation (13) was constructed in the papers [23] - 24]. Let us present the data from this theory which will be necessary further.

\section{Definition 4}

Operators $\hat{X}$ and $\hat{X}^{\prime}$ are equivalent according to the function $F$, if:

$$
\hat{X}^{\prime}=\exp (-F) \hat{X} \exp F .
$$




\section{Definition 5}

The equation (13) admits a complete separation of variables provided there are function $F$ and the privileged coordinate system $\left\{u^{i}\right\}$ that allow to represent the solution of the equation

$$
\hat{H}^{\prime} \phi=\lambda \phi
$$

into the following form:

$$
\phi=\prod_{i} \varphi_{i}\left(u^{i} \lambda_{j}\right), \quad \operatorname{det}\left\|\frac{\partial^{2} \phi}{\partial u^{i} \partial \lambda_{j}}\right\| \neq 0 ;
$$

where $\lambda_{i} \quad$ essential parameters $\quad\left(\lambda_{n-1}=\lambda\right)$. The solution to the equation (13) is represented with $\phi$ and $F$ as follows:

$$
\Phi=\exp F(u) \phi
$$

\section{Theorem 3}

The equation (13) admits the complete separation of variables if and only if there is a complete set of mutionally-commuting symmetry operators.

$$
\hat{X}_{p}=Y_{p}^{i} \hat{\partial}_{i}, \quad \hat{X}_{\nu}=X_{\nu}{ }^{i j} \hat{\partial}_{i} \hat{\partial}_{j}+2 X_{\nu}{ }^{i} \hat{\partial}_{i}+X_{\nu}, \quad\left(\left[\hat{X}_{i}, \hat{X}_{j}\right]=0, \quad \hat{X}_{n-1}=\hat{H}\right) .
$$

The essential parameters $\lambda_{i}$ introduced above are eigenvalues, and the function $\Phi$ is the eigenfunction of the operators $\hat{X}_{i}$ :

$$
\hat{X}_{i} \Phi=\lambda_{i} \Phi .
$$

Obviously, the functions $X_{\nu}^{i j}, Y_{p}^{i}$ form a complete set in according to the Definition 2.

Note that the condition of mutually commutativity of the symmetry operators of the equation (13) is not necessary for the exact integration, since the theory of Stackel spaces can be generalized for a case of non-commutative operators of motion (see, 25] [26]).

Theorem 4 If the equation (13) admits complete separation of variables in the privileged coordinate system $\left\{u^{l}\right\}$ in the same coordinate system the equation (11) admits complete separation of variables too. Thus the $V_{n}$ belongs to the Stackel space

A theorem similar to Theorem 2 is valid:

\section{Theorem 5}

If the equation (13) admits a complete separation of variables the complete set of the symmetry operators (15) can be presented as:

$$
\hat{X}_{q}=\hat{\partial}_{q}, \quad \hat{X}_{\mu}^{\prime}=\left(\hat{\phi}^{-1}\right)_{\mu}^{\nu} \hat{H}_{\nu} .
$$

where

$$
\hat{H}_{\nu}=\varepsilon_{\nu} \hat{\partial}_{\nu}^{2}+h_{\nu}^{p q} \hat{\partial}_{p} \hat{\partial}_{q}+2 h_{\nu}^{\nu q} \hat{\partial}_{\nu} \hat{\partial}_{q}+2 h_{\nu}^{q} \hat{\partial}_{q}+2 h_{\nu}^{\nu} \hat{\partial}_{\nu}+h_{\nu}
$$


Klein-Gordon-Fock equation is a particular case of the equation (13):

$$
\hat{H} \Phi=\left(-\imath \hat{\nabla}^{i}+A^{i}\right)\left(-\imath \hat{\nabla}_{i}+A_{i}\right) \Phi=\lambda \Phi,
$$

Using the metrics of Stackel spaces one can represent the equation (17) in the form:

$$
\left[G^{i j} \hat{\partial}_{i} \hat{\partial}_{j}+\left(G_{, i}^{i j}+G^{i j} \chi_{, i}+2 \imath B^{j}\right) \hat{\partial}_{, j}+\imath\left(B_{, i}^{i}+\chi_{, i} B^{i}\right)-B^{i} A_{i}+\lambda \Delta\right] \Phi=0,
$$

where:

$$
G^{i j}=g^{i j} \Delta, \quad B^{i}=A^{i} \Delta, \quad G=\operatorname{det}\left\|G^{i j}\right\|, \quad \chi=\frac{1}{2}\left(\ln \frac{\Delta^{n-2}}{G}\right), \quad \Delta=\operatorname{det}\left\|\varphi_{\mu}^{\nu}\right\| .
$$

We will consider the equation (18) provided that the Hamilton-Jacobi equation for a charged particle admits complete separation of variables. Then the relations (6) are true. In addition, we assume that the following condition is satisfied:

$$
\chi=\ln \alpha_{1} \alpha_{2} \alpha_{3} \quad \rightarrow \Delta^{2}=G \alpha_{1} \alpha_{2} \alpha_{3} .
$$

In this case the function $F=1$, and equation (18) admits complete separation of variables.

\subsection{Solution of the functional equation}

For the space-time of type (1.1) with metric tensor (12) the operator $\hat{H}$ can be written in the form:

$$
\hat{H}=\frac{V^{\alpha} \hat{H}_{\alpha}}{\Delta}
$$

where

$$
\begin{gathered}
\hat{H}_{1}=2\left(\hat{\partial}_{0}+\imath \xi e_{1}\right) \hat{\partial}_{1}+\dot{\alpha}_{1}\left(\hat{\partial}_{0}+\imath \xi_{0} e_{1}\right)+\imath \xi_{0} \dot{e}_{1}, \\
\hat{H}_{\nu}=\varepsilon_{\nu}\left(\hat{\partial}_{\nu}^{2}+\dot{\alpha}_{\nu} \hat{\partial}_{\nu}\right)+\xi_{1}\left(b_{\nu} \hat{\partial}_{0}^{2}+2 \imath a_{\nu} \hat{\partial}_{0}\right) .
\end{gathered}
$$

Symmetry operators have the form:

$$
\begin{aligned}
& \hat{X}_{1}=\frac{\left(\tau_{2} \omega_{3}-\tau_{3} \omega_{2}\right) \hat{H}_{1}+\left(\tau_{3} \omega_{1}-\tau_{1} \omega_{3}\right) \hat{H}_{2}+\left(\tau_{1} \omega_{2}-\tau_{2} \omega_{1}\right) \hat{H}_{3}}{\Delta}, \\
& \hat{X}_{2}=\frac{\left(\omega_{2}-\omega_{3}\right) H_{1}+\left(\omega_{3}-\omega_{1}\right) H_{2}+\left(\omega_{1}-\omega_{2}\right) H_{3}}{\Delta}, \quad \hat{X}_{0}=\delta_{0}^{i} \hat{\partial}_{i} .
\end{aligned}
$$

Then the separated system can be represented as:

$$
\begin{aligned}
\imath\left[2\left(\lambda_{0}+\xi_{0} e_{1}\right) \hat{\partial}_{1}+\dot{\alpha}_{1}\left(\lambda_{0}-e_{1} \xi_{0}\right)+\xi_{0} \dot{e}_{1}\right] \varphi_{1} & =\left(\lambda_{1}+\lambda_{2} \tau_{1}+\lambda_{3} \omega_{1}\right) \varphi_{1}, \\
{\left[\varepsilon_{\nu}\left(\hat{\partial}_{\nu}^{2}+\dot{\alpha}_{\nu} \hat{\partial}_{\nu}\right)-\xi_{1}\left(b_{\nu} \lambda_{0}{ }^{2}+2 a_{\nu} \lambda_{0}\right)\right] \varphi_{\nu} } & =\left(\lambda_{1}+\lambda_{2} \tau_{\nu}+\lambda_{3} \omega_{\nu}\right) \varphi_{\nu} .
\end{aligned}
$$


The functional equation (19) has the form:

$$
\frac{\Delta}{\alpha_{1} \alpha_{2} \alpha_{3}\left(\tau_{2}-\tau_{3}\right)}=\sqrt{\varepsilon\left(\tau_{2}-\tau_{1}\right)\left(\tau_{3}-\tau_{1}\right)} .
$$

The left side of the equation is a rational function containing functions $\left(\alpha_{\alpha}, \tau_{\alpha}, w_{\alpha}\right)$, each of them depends on one variable. The right side is an irrational function. Therefore the equation has solutions provided that

$$
\dot{\tau}_{1}\left(\dot{\tau}_{2}^{2}+\dot{\tau}_{3}^{2}\right)=0
$$

Let us consider the function equation (3.1) for all options.

I. $\dot{\tau}_{1}=0$. The relations (12) can be presented as follows:

$$
\begin{aligned}
\hat{g}^{i j}= & \left(\begin{array}{cccc}
\xi_{1} \frac{b_{2}+b_{3}}{\Delta} & \frac{\tau_{2}+\tau_{3}}{\Delta} & 0 & 0 \\
\frac{\tau_{2}+\tau_{3}}{\Delta} & 0 & 0 & 0 \\
0 & 0 & \frac{1}{\Delta} & 0 \\
0 & 0 & 0 & \frac{1}{\Delta}
\end{array}\right), \quad A^{i}=\frac{\xi_{1} \delta^{i 0}\left(a_{2}+a_{3}\right)+\xi_{0} \delta^{i 1} e_{1}\left(\tau_{2}+\tau_{3}\right)}{\Delta} \\
\Delta & =w_{1}\left(\tau_{2}+\tau_{3}\right)+w_{2}+w_{3} .
\end{aligned}
$$

It allows to represent the functional equation in the form:

$$
w_{1}\left(\tau_{2}+\tau_{3}\right)+w_{2}+w_{3}=\alpha_{1} \alpha_{2} \alpha_{3}\left(\tau_{2}+\tau_{3}\right) .
$$

Let us consider possible options.

a. $\quad \dot{\alpha}_{1}=0$, which is equivalent to $w_{1}=0$. Solution of the equation (23) has the form:

$$
\Delta=\frac{\left(\tau_{2}+\tau_{3}\right)}{\tau_{2} \tau_{3}} \quad\left(\alpha_{1}=1, \quad \alpha_{\nu}=\frac{1}{\tau_{\nu}}\right) .
$$

b. $\quad \dot{\alpha}_{1} \neq 0 . \quad \rightarrow w_{1}=\alpha_{1}$, and from the equation (23) it follows:

$$
\alpha_{2} \alpha_{3}=1, \quad w_{\nu}=0 \quad \rightarrow \quad \Delta=\alpha_{1}\left(\tau_{2}+\tau_{3}\right)
$$

II. $\quad \dot{\tau}_{1} \neq 0 \rightarrow \tau_{\nu}=(-1)^{\nu}, \quad \dot{w}_{2} \dot{w}_{3}=0$. The relations (12) can be presented as follows:

$$
\begin{gathered}
\hat{g}^{i j}=\left(\begin{array}{cccc}
\xi_{1} \frac{b_{2}\left(\tau_{1}-1\right)+b_{3}\left(\tau_{1}+1\right)}{\Delta} & \frac{1}{\Delta} & 0 & 0 \\
\frac{1}{\Delta} & 0 & 0 & 0 \\
0 & 0 & \frac{\varepsilon_{2}\left(\tau_{1}-1\right)}{\Delta} & 0 \\
0 & 0 & 0 & \frac{\varepsilon_{3}\left(\tau_{1}+1\right)}{\Delta}
\end{array}\right), \\
A^{i}=\frac{\xi_{1} \delta^{i 0}\left(a_{2}\left(\tau_{1}-1\right)+a_{3}\left(\tau_{1}+1\right)\right)+\xi_{0} \delta^{i 1} e_{1}}{\Delta}, \\
\Delta=w_{1}+w_{2}\left(\tau_{1}-1\right)+w_{3}\left(\tau_{1}+1\right) .
\end{gathered}
$$


The following options are possible:

a. $\quad w_{3}=0, \quad \dot{w}_{2} \neq 0 . \quad$ The solution to the equation (3.1) has the form:

$$
\Delta=\left(\tau_{1}-1\right) \exp \alpha_{2} .
$$

b. $\quad w_{\nu}=0$ From (23) it follows:

$$
\Delta=\alpha_{1}
$$

\subsection{Results}

Let us list all sets containing the obtained metrics and electro-magnetic potentials, for which the Kleyn-Gordon-Fock equation (18) admits the complete separation of variables. The function

$$
\Phi=\varphi_{1} \varphi_{2} \varphi_{3} \exp \imath \lambda_{0} u^{0}
$$

is an eigenfunction of the symmetry operators $\hat{X}_{i}$ with eigenvalues $\lambda_{i}$ :

$$
\hat{X}_{i} \Phi=\lambda_{i} \Phi
$$

\section{Set1.}

Metric:

$$
d s^{2}=\left(2 d u^{0} d u^{1}-d u^{0^{2}} \xi_{1} \frac{b_{2}+b_{3}}{\tau_{2}+\tau_{3}}+\left(\tau_{2}+\tau_{3}\right)\left(d u^{2^{2}}+d u^{3^{2}}\right)\right) \exp \beta_{1} .
$$

Electro-magnetic potential:

$$
A_{i}=\xi_{1} \delta_{i 1} \frac{a_{2}+a_{3}}{\tau_{2}+\tau_{3}}+\xi_{0} \delta_{i 0} e_{1}
$$

\section{Set 2 .}

\section{Metric:}

$$
d s^{2}=\frac{1}{\tau_{2} \tau_{3}}\left(2 d u^{0} d u^{1}-d u^{12} \xi_{1} \frac{b_{2}+b_{3}}{\tau_{2}+\tau_{3}}+\left(\tau_{2}+\tau_{3}\right)\left(d u^{2^{2}}+d u^{3^{2}}\right)\right) .
$$


Electro-magnetic potential:

$$
A_{i}=\xi_{1} \delta_{i 1} \frac{a_{2}+a_{3}}{\tau_{2}+\tau_{3}}+\xi_{0} \delta_{i 0} e_{1}
$$

Set 3 .

Metric:

$$
\begin{array}{r}
d s^{2}=2 d u^{0} d u^{1}-d u^{1^{2}} \xi_{1}\left(b_{2} \exp \beta_{1}+b_{3} \exp \gamma_{1}\right)+d u^{2^{2}} \exp -\beta_{1}+ \\
d u^{3^{2}} \exp -\gamma_{1}
\end{array}
$$

Electro-magnetic potential:

$$
A_{i}=\xi_{0} \delta_{i 0} e_{1}+\xi_{1} \delta_{i 1}\left(a_{2} \exp \beta_{1}+a_{3} \exp \gamma_{1}\right) .
$$

Set 4 .

Metric:

$$
d s^{2}=\left(2 d u^{0} d u^{1}-d u^{1^{2}} \xi_{1}\left(b_{2}+b_{3} \exp \gamma_{1}\right)+d u^{2^{2}}+d u^{3^{2}} \exp -\gamma_{1}\right) \exp \beta_{2} .
$$

Electro-magnetic potential:

$$
A_{i}=\xi_{0} \delta_{i 0} e_{1}+\xi_{1} \delta_{i 1}\left(a_{2}+a_{3} \exp \gamma_{1}\right) .
$$

\section{Conclusion}

The main result of this work is the final solution to the classification problem considered in the papers [1]-[3]. Thus all Stackel space-time metrics and electro-magnetic potentials of the external electromagnetic field for which the Hamilton-Jacobi equation admits complete separation of variables were found. From a physical point of view, attention to external fields is due to the presence of physical processes, in the study of which the influence on the geometry of all fields except the gravitational one can be neglected. As an example, we will present axion fields, which are currently considered as the closest candidates for describing dark matter. When constructing cosmological models of the early Universe, they make a significant contribution to the energy-momentum tensor and therefore cannot be considered as external. [27]-[29]. At the same time, when studying a set of local effects in strong gravitational fields, the electromagnetic and axion fields, due to their insignificant contribution to the formation of space-time geometry, often need to be considered 
just as external against the background of a given metric. Thus, in [30], the axion, and electromagnetic fields are related by the following system of equations:

$$
\nabla_{i}\left(F^{i j}+\Phi F^{* i j}\right)=0, \quad g^{i j} \nabla_{i} \nabla_{j} \Phi+\frac{1}{2} \frac{\partial V(\Phi)}{\partial \Phi}=-\tilde{\kappa}^{2} F^{* i j} F_{i j},
$$

where $F^{* i j}$ is the electromagnetic field tensor dual to $F_{i j}, g^{i j}$ is a given metric tensor. In some local problems, the function $V$ can be considered null and the second equation of the system is a linear inhomogeneous equation with respect to the function $\Phi$. The problem of classifying the Stackel metrics, electromagnetic potentials, and the scalar field satisfying the system of equations (28) seems to be quite interesting from the physical point of view.

We note two more promising areas of application of Stackel spaces theory in the theory of gravity.

1) In multidimensional theories of gravity. The interest in multidimensional spaces is due to the emergence of generalizations of the Kaluza-Klein theory, as well as superstring theory. Large number of papers have been devoted to the problems of multidimensional gravity (see, for example, [31] - [34]).

2) As already noted, among the Stackel space-times of type (N.1) there are wave-like ones (see article [35] too). Recently, interest in wave metrics has increased significantly. For example, in the papers [36]-[37] propagation of gravitational waves in accelerating universe was described. The results obtained in our paper allow us to construct realistic models with gravitational and electromagnetic plane waves.

These tasks are supposed to be considered in the future.

Acknowledgment.

This work was supported by the Ministry of Science and Higher Education of the Russian Federation, project FEWF-2020-0003.

\section{References}

[1] V.V. Obukhov, Integration of the Hamilton-Jacobi and Maxwell equations for Diagonal metrics. Russ. Phys. J. 63, (2020), N 7, 3335.

[2] V.V.Obukhov, Hamilton-Jacobi equation for a charged test particle in the Stackel space of type (2.0). Symmetry, 12, (2020) 12891291. 
[3] V.V.Obukhov, Hamilton-Jacobi equation for a charged test particle in the Stackel space of type (2.1). IJGMMP 17, (2020), N 14, 2050186 (20 pages)

[4] P. Stackel, Uber die intagration der Hamiltonschen differentialechung mittels separation der variablen. Math. Ann. 49, (1897), 145-147.

[5] T. Levi-Civita. Sulla integraziome della Equazione di HamiltonJacobi per separazione di variabili. Math.Ann. 59, (1904), 383-397.

[6] M.S.Jarov-Jrovoy. Integration of Hamilton-Jacobi equation by complete separation of variables method. J.Appl.Math.Mech. 27, (1963), N 6, 173-219.

[7] V.N. Shapovalov, Symmetry and separation of variables in the Hamilton-Jacobi equation. Sov. Phys.J. 21, (1978), 1124-1132.

[8] V.N. Shapovalov, Stackel's spaces. Sib. Math. J. 20, (1979), 1117-1130.

[9] V.G. Bagrov, V.V. Obukhov, Complete separation of variables in the free Hamilton-Jacobi equation. TMF. 97, (1993) N 2, (1275-1289).

[10] S. Benenti, Separability in riemannian manifolds. Symmetry, integrability and geometry: methods and applications, Sigma. 12, (2016), 013.

[11] W. Miller. Symmetry and separation of variables. (Cambridge University Press: Cambridge, 1984).

[12] S. Benenti, C. Chanu, G.Rastelli, Variables separation for natural Hamiltonians with scalar and vector potentials on Riemannian manifolds. Math. Phis. 42, (2001), N 5, 2065-2091.

[13] Bagrov, A. G. Meshkov, V. N. Shapovalov, A. V. Shapovalov, Separation of variables in the Klein-Gordon equations I. Sov. Phys.J. (1973), N 6, 1533-1538.

[14] Bagrov, A. G. Meshkov, V. N. Shapovalov, A. V. Shapovalov, Separation of variables in the Klein-Gordon equations II. Sov. Phys.J. (1973), N 16, 1659-1665.

[15] V. G. Bagrov, A. G. Meshkov, V. N. Shapovalov, A. V. Shapovalov, Separation of variables in the Klein-Gordon equations III. Sov. Phys. J. (1974), N 17, 812-815.

[16] V.G. Bagrov, A.V. Obukhov, Classes of exact solutions of the Einstein-Maxwell equations. Ann. der Phys. B40, (1983), H 4/5, 181188.

[17] V.G. Bagrov, V.V. Obukhov, A.V. Shapovalov, Special Stackel electrovac spacetimes, Pramana J. Phys. 26 (1986) N 2, 93-108. 
[18] V. V. Obukhov, K. E. Osetrin, A. E. Filippov, Yu. A. Rybalov, The Vaidya problem in conformally flat Stackel spaces of type (1.1). Russ. Phys. J. 52, (2009) N 1, 11-14. DOI: 10.1007/s11182-009-9198-3.

[19] V. G. Bagrov, V. V. Obukhov, A. V. Shapovalov. Gravitation field in the Vaidya problem allowing separation of variables in the Hamilton-Jacobi equation. Sov. Physics J. 29, (1986), N 10, 775-779. DOI: $10.1007 /$ BF00900736.

[20] E. Osetrin and K. Osetrin, Pure radiation in space-time models that admit integration of the eikonal equation by the separation of variables method. J. Math. Phys. 58, (2017), N 11, 112504.

[21] K.E. Osetrin, A.E. Filippov and E.K. Osetrin, Models of generalized scalar-tensor gravitation theories with radiation allowing the separation of variables in the eikonal equation. Russ. Phys. J. 61, (2018), N 8, 1383-1391.

[22] K. Osetrin, A. Filippov and E. Osetrin, The spacetime models with dust matter that admit separation of variables in Hamilton-Jacobi equations of a test particle. Modern Phys. Lett. A, 31, (2016), N 6, 1650027.

[23] V.N. Shapovalov, Symmetry and separation of variables in a linear second-order differential equation. I. Sov. Phys.J. (1978), N 21, 645650.

[24] V.N. Shapovalov, Symmetry and separation of variables in a linear second-order differential equation. II. Sov. Phys.J., (1978), N 21, 693-695.

[25] A.V. Shapovalov, I.V. Shirokov. Non-commutative integration of linear Differential equations. $T M F$. 104, (1995), N 2, 195-213.

[26] A. Breev, A. V. Shapovalov, Non-commutative integration of the Dirac tquation in homogeneous spaces. Symmetry, 12, (2020), 1867.

[27] S. Nojiri, S.D. Odintsov, V.K. Oikonomou, $F(R)$ Gravity with an axion-like particle: dynamics, gravity waves, late and early-time phenomenology. Annals Phys. 418, (2020), 168186.

[28] S.D. Odintsov, V.K. Oikonomou, Aspects of axion $F(R)$ gravity. $E P L$, 129, (2020), N 4, 40001.

[29] I.V. Fomin, S. V. Chervon, Exact and approximate solutions in the Friedmann cosmology. Russ. Phys. J. 60, (2017), N 30, 427-440.

[30] Balakin A.B. The Extended Einstein-Maxwell-Aether-Axion Model: exact solutions for axionically controlled Pp-Wave aether modes. Modern Physics Letters A. 2018, (2018), N 33, 1850050. 
[31] K.K. Ernazarov, V.D. Ivashchuk, Stable exponential cosmological solutions with zero variation of $\mathrm{G}$ in the Einstein - Gauss - Bonnet model with a $\Lambda$-term. Eur. Phys. J. C. (2017), 77: 89.

[32] V. D. Ivashchuk, On stability of exponential cosmological solutions with non-static volume factor in the Einstein-Gauss-Bonnet model. Eur. Phys. J. C, (2016), 76:431.

[33] V. P. Frolov, P. Krtous Charged particle in higher dimensional weakly charged rotating black hole spacetime Phys. Rev.D, 83, (2011), 024016.

[34] Ya. V. Lisitsyn, A. V. Shapovalov, Integrable N-dimensional systems on the Hopf algebra and Q-deformations. TMP. 124:3, (2000), 1172.

[35] K.Osetrin, E.Osetrin, Shapovalov wave-like spacetimes. Symmetry, 12, (2020), 1372.

[36] S. Capozziello, M. De Laurentis, S. Nojiri, S.D. Odintsov, Evolution of gravitons in accelerating cosmologies: The case of extended gravity. Phys.Rev.D, 95, (2017), N 8, 083524,

[37] K. Bamba, S. Nojiri, S. D. Odintsov, Propagation of gravitational waves in strong magnetic fields. Phys.Rev. D. 98, (2018), N 2, 024002. 